\title{
GESTIÓN DE RECURSOS HUMANOS EN LAS MIPYMES DE COMUNICACIONES Y TRANSPORTES EN EL ESTADO DE TABASCO
}

\author{
Wendy Vanessa Oropeza Ruiz * Emma Patricia Santana Martínez**
}

\begin{abstract}
Oropeza-Ruíz W.V., Santana-Martínez E.P. Gestión de recursos humanos en las MIPYMES de comunicaciones y transportes en el Estado de Tabasco. Hitos de Ciencias Económico Administrativas 2013;19 (55): 131-138.
\end{abstract}

RESUMEN

Objetivo: Conocer la gestión de recursos humanos que influye en el éxito competitivo de las MIPYMES de comunicaciones y transportes del estado de Tabasco y cómo se encuentra con respecto a otros estados del país.

Material y método: El estudio fue dirigido a las MIPYMES de los subsectores de comunicaciones y transportes del estado de Tabasco y los resultados se obtuvieron de una muestra representativa de 81 empresas, mismas que fueron analizadas por tamaño y antigüedad. Como marco muestral se tomó el censo económico del INEGI 2004, posteriormente actualizado con el censo económico 2009, del cual se determinó la muestra de los subsectores, por medio de un muestreo aleatorio sistemático. La recolección de datos se llevó a cabo a través de un cuestionario diseñado ex profeso utilizado en las entrevistas. Para la comparación con otros estados del país, se tomaron los resultados de estudios análogos al presente.

Resultados: La práctica menos utilizada en las MIPYMES de los subsectores estudiados es la promoción de personal, sin importar el tamaño y la antigüedad de la empresa; y la más aplicada es la capacitación del personal. Se ha comprobado que los procesos de gestión de recursos humanos de las MIPYMES de comunicaciones y transportes del estado de Tabasco son bajos en comparación con otros estados del país tomados como referencia.

\author{
Oropeza-Ruíz W.V., Santana-Martínez E.P. \\ Human resource management in the communications \\ and transportation MSMEs in the state of Tabasco. \\ Hitos de Ciencias Económico Administrativas \\ 2013;19 (55): 131-138.
}

\section{ABSTRACT}

Objective: To know the human resources management that influences the competitive success of the Communications and Transportation MSMEs in the state of Tabasco and what is its status regarding to other states in the country.

Material and method: The study was aimed to the MSMEs of the communications and transportation subsectors in the state of Tabasco, and the results were obtained from a representative sample of 81 companies which were analyzed by size and age. As a sampling frame, the INEGI 2004 economic census was considered, afterwards, it was updated with the 2009 Economic Census, and from this last one it was determined the sample of the subsectors by means of a systematic random sampling. The data gathering was held through an expressly designed questionnaire used in the interviews. For the comparisons with other states in the country, the results of similar studies were taken.

Results: The least used practice in the MSMEs of the studied subsectors is the promotion of personnel, regardless the size and age of the company, and the most used practice is the training of the personnel. It has been found that the processes of human resources management in the SMEs of the communications and transportation subsectors in Tabasco are low compared to other states that were taken as reference.

*Maestra en Administración. División Académica de Ciencias Económico Administrativas. Universidad Juárez Autónoma de Tabasco.

**Maestra en Administración. División Académica de Ciencias Económico Administrativas. Universidad Juárez Autónoma de Tabasco.

Fecha de recibido: 12 de febrero de 2013 Fecha de aceptación: 14 de marzo de 2013. 
Conclusiones: Las prácticas de gestión de recursos humanos de las empresas estudiadas son poco utilizadas y no se relacionan entre sí. Por lo anterior se considera necesaria la mayor aplicación de las actividades de gestión de recursos humanos dentro de las MIPYMES tabasqueñas de los subsectores estudiados, así como la sinergia entre ellas para la eficiencia en el proceso y en el capital humano.
Conclusions: The practices of human resources management of the studied companies are rarely used and are not related among them. Therefore, it is important to consider necessary the implementation of more of the activities of the human resources management within the MSMEs in Tabasco in the studied subsectors, as well as the synergy among them for the efficiency in the process and in human capital.
Palabras claves: Micro, Pequeña y Mediana Empresa, Gestión de Recursos Humanos, Comunicaciones y Transportes.
Key words: Micro. Small and Medium Enterprises. Human Resource Management. Communications and Transportation.

DIRECCIÓN PARA RECIBIR CORRESPONDENCIA: Correo electrónico: Wendy_Vane88@hotmail.com

a micro, pequeña y mediana empresa (MIPYMES) ha sido en los últimos años el centro de atención de numerosos trabajos, debido fundamentalmente a su gran capacidad de generación de empleo, así como el papel primordial que juegan como generadoras de riqueza (Chablé y Aragón, 2009).

En Tabasco el sector empresarial suma, según el censo económico de 2009 , un total de 52,663 negocios, en donde las microempresas (hasta 10 empleados) constituyen el $94.3 \%$, le siguen en importancia las pequeñas (de 11 a 50 ) con el $4.6 \%$ de la representación total, el $1 \%$ de medianas (de 51 a 250 ) y el resto de grandes.

No obstante, las MIPYMES siguen necesitadas de fundamentos operativos que, de forma continua, pongan de manifiesto su problemática y sus estrategias al objeto de facilitar la toma de decisiones, tanto desde un punto de vista de política interna de la empresa para su gestión, como de política local o estatal, para determinar y fijar programas de actuación acertados y oportunos.

Es por ello que el análisis de la gestión de los recursos humanos, como factor de competitividad dentro de las MIPYMES, en este caso de comunicaciones y transportes, es importante y necesario; ya que las debilidades de carácter estructural que padecen las MIPYMES aunado al escaso hábito de gestión del conocimiento y del capital humano, la menos calificación de empleados, entre otros factores, siguen afectando la supervivencia de éstas y mermando en su competitividad.

\section{Marco teórico}

Los recursos humanos son factor importante para el desarrollo y crecimiento de las empresas en un mercado globalizado como en el que en la actualidad se desenvuelven. Ya que «el capital humano constituye actualmente uno de los factores determinantes para la obtención de valor agregado. Este valor se potencia cuando el conocimiento se coloca en función del logro de los objetivos de la organización. El capital humano depende en gran medida de la capacidad de las organizaciones para desarrollar y aprovechar el conocimiento» (Hernández \& Martí, 2006).

«Para lograr el éxito competitivo, las empresas deben establecer los mecanismos que permitan atraer candidatos cualificados, retener y motivar a los actuales empleados y establecer fórmulas que los ayuden a crecer y desarrollarse dentro de la empresa. En definitiva, han de prestar gran atención a aquellas prácticas que favorezcan el desarrollo del capital humano y permitan gestionar correctamente el conocimiento organizativo. No reconocer el valor requerido a la gestión de los recursos humanos es una de las principales causas de fracaso en las PYMES» (Rubio \& Aragón, 2006).

En la misma dirección Hernández \& Martí (2006) señala que la gestión de recursos humanos tiene una gran importancia para las organizaciones, debido a la sinergia en el desarrollo integral de políticas dirigidas a un mejor desempeño de los recursos humanos mediante la ejecución de diversas actividades. Este proceso permite a las organizaciones reorientarse en función de las debilidades y fortalezas de los activos intangibles. 
Uno de los elementos de la gestión de los recursos humanos que tributa al mejoramiento del desempeño y a la disminución de las debilidades de los activos intangibles es la capacitación.

Shaun (2004), establece que la capacitación sólo puede tener un propósito general y terminal, el cual consiste en permitir que el trabajo se pueda realizar de un modo efectivo en tres niveles: por la organización como un todo, en los subgrupos que lo componen y por sus empleados individuales.

Otra práctica de recursos humanos es el reclutamiento y selección de personal, siendo para Chiavenato (2000) el reclutamiento un conjunto de técnicas y procedimientos orientado a atraer candidatos potencialmente calificados y capaces de ocupar cargos dentro de la organización; y «la selección de personal escoger entre los candidatos reclutados los más adecuados, para ocupar los cargos existentes en la empresa.»

La finalidad de la selección del personal es definir políticas y prácticas de obtención del personal idóneo a la organización, así como seleccionar el personal, sea para incorporarlo a la organización, para su promoción o para su capacitación. Es un proceso permanente, planificado y es responsabilidad de todas las áreas funcionales, aunque se dirija desde recursos humanos. Dicha promoción de personal debe posibilitar la plena realización personal de cada trabajador de la empresa así como su crecimiento dentro de la misma, según sus capacidades e intereses (Hernández \& Martí, 2006).

Un elemento que sin duda contribuye en la motivación del personal para que éste sea más eficiente y productivo en sus tareas, son las recompensas o incentivos otorgados a los trabajadores, y tales recompensas varían dependiendo de cada institución, y pueden ser incentivos económicos o no económicos. Chiavenato (2000), señala que éstos «deben ser adecuados, equitativos, eficientes en costos, seguros y aceptables para los trabajadores. De esta manera, se alcanzará el equilibrio y éxito de la organización en cuanto a políticas de remuneración y planes de incentivos, los cuales se ven fortalecidos cuando se considera al trabajador como el elemento fundamental de la misma».
Sin embargo, dicho programa de incentivos no es posible sin realizar otro elemento primordial: la evaluación del desempeño que es una apreciación sistemática del desempeño de cada persona en el cargo o del potencial de desarrollo futuro (Chiavenato, 2000). Cabe mencionar que los programas de incentivos mantienen gran importancia en el área de la administración de recursos humanos, tomando en cuenta que el capital humano se considera como el elemento fundamental del funcionamiento de cualquier organización.

«Newstrom (2007) señala que el alto desempeño contribuye a una alta satisfacción en el trabajo, un mejor desempeño suele conducir a mayores recompensas económicas, sociológicas y psicológicas. Si éstas se consideran justas y equitativas, entonces mejorará el nivel de satisfacción porque los empleados sienten que reciben estímulos en proporción a su desempeño. Por otro lado, si las recompensas se consideran inadecuadas para el nivel de desempeño, la insatisfacción tenderá a elevarse.

Carrasco \& Rubio (2007) demostraron que la Pyme familiar exitosa es aquella que utiliza unas prácticas de recursos humanos más acordes con las exigencias del mercado, es decir, prácticas más profesionalizadas o «mejores prácticas». Por su parte, las pymes no exitosas emplean prácticas más tradicionales, menos formales.

\section{Material y método}

El tipo de investigación que se realizó fue: aplicada, documental y de campo In situ, no experimental o expost-facto y descriptivo y correlacional. El enfoque fue cuantitativo y cualitativo. La población objeto de estudio está conformada por micro, pequeñas y medianas empresas de los subsectores comunicaciones y transportes del estado de Tabasco. El criterio de selección fue mediante un muestreo aleatorio sistemático, clasificando a las empresas por tamaños según el número de empleos generados: microempresas (más de 4 y menos de 11 trabajadores), pequeñas empresas (de 11 a 50 trabajadores) y empresas medianas (de 51 a 250 trabajadores); y como marco muestral se tomó el censo económico levantado por el INEGI con datos de 2004, actualizando posteriormente con el censo económico 2009 cuando se tuvo disponible. 
La fórmula para determinar el tamaño de la muestra que se utilizó es la siguiente:

Diseño estadístico: $\quad n_{i}=\frac{4 p q N}{\left[\varepsilon^{2}(N-1)+4 p q\right]}$

En donde:

$\mathbf{n}_{\mathbf{i}}=$ al tamaño de la muestra a estimar de los subsectores; $\mathbf{p}$ y q son las proporciones de respuestas dicotómicas que para mejor representatividad se considera $0.5 ; \mathbf{N}=$ es la población del universo de los subsectores de empresas censadas que resultó de 428; e = error de precisión adoptado, para cada caso fue el máximo del $10 \%$; cálculo que arrojó un total de 81 encuestas a levantar (suma de las $n_{i}$ ) como se muestra a continuación:

$n_{i}=\frac{4(0.5)(0.5) 428}{\left[(0.10)^{2}(428-1)+4(0.5)(0.5)\right]}=\frac{4(428)}{\left[(0.01)^{2}(427)+1\right]}=\frac{428}{[5.227]}=81.21$

El tamaño de la muestra determinado es de 81 empresas. La recopilación de información fue mediante entrevistas, en base a una encuesta, a los directores o gerentes de las MIPYMES seleccionadas.

El objetivo general es conocer la gestión de recursos humanos que influye en el éxito competitivo de las MIPYMES de los subsectores comunicaciones y transportes del estado de Tabasco y cómo se encuentra con respecto a otros estados del país.

Las hipótesis para las contrastaciones estadísticas de la investigación que se plantearon fueron: Hipótesis nula que considera a los procesos de gestión de recursos humanos de las MIPYMES de comunicaciones y transportes en el estado de Tabasco semejantes al de otros estados del país tomados como referencia.

Las hipótesis secundarias se hicieron con respecto a las siguientes cinco variables: el reclutamiento y selección del personal, valoración del desempeño, capacitación del personal, promoción del personal y programa de incentivos. Considerando la aplicación de éstas en las MIPYMES de los subsectores comunicaciones y transportes en el estado de Tabasco en un nivel bajo en comparación con otros estados del país tomados como referencia.

En la obtención de los tamaños de las muestras de los estados que se toman como referencia en esta investigación, el procedimiento que se siguió fue tomar los resultados de estudios análogos sobre las Micro, Pequeñas y Medianas empresas de los subsectores comunicaciones y transportes del sector terciario de los estados donde ya se han realizado investigaciones con la misma metodología, como son: Aguascalientes, Nayarit, Durango y Veracruz.

El estadístico de prueba que se utilizó para contrastar las hipótesis de trabajo fue la diferencia de proporciones para cada variable especificada en las hipótesis secundarias, al comparar a Tabasco con otros estados del país tomados como referencia (Aguascalientes, Nayarit, Veracruz y Durango).

Cabe mencionar que este estudio, trata de probar, por medio del uso de cada variable antes mencionada que la práctica del proceso de gestión de recursos en el estado de Tabasco es baja con respecto a los estados de referencia (Aguascalientes, Durango, Nayarit y Veracruz). Por lo tanto, así se plantean las hipótesis estadísticas alternativas y se les graficó sobre la distribución Z como se muestra en la figura 1, con una sola región de rechazo en la zona izquierda. 
En consecuencia necesitábamos para la prueba de hipótesis entre dos proporciones una $n_{p}$ la cual obtuvimos como sigue:

Sumamos todas las muestras (MIPYMES de los subsectores comunicaciones y transportes) de los estados: Durango (25), Nayarit (11), Aguascalientes (8) y Veracruz (36), y obtenemos $\mathbf{n p}=\mathbf{8 0}$.

\section{Resultados}

Las características de las empresas que conforman esta investigación indican en cuanto al tamaño que $40.7 \%$ son microempresas, $43.2 \%$ pequeñas empresas y $16.1 \%$ medianas empresas. Con respecto a la antigüedad, $24.7 \%$ son empresas que tienen 10 años o menos dentro del mercado, mientras $75.3 \%$ restante tiene más de 10 años operando.

Como se observa en la figura 2, la práctica de recursos humanos más aplicada es la de capacitación de personal, seguida por el reclutamiento y selección de personal; sin embargo la promoción de personal muestra el menor porcentaje de aplicación, lo cual refleja la poca oportunidad de crecimiento de los empleados dentro de las organizaciones.

Partiendo de los datos anteriores y con respecto a las prácticas de gestión de los recursos humanos estudiadas los datos muestran (figura 3 ) que en el caso del reclutamiento y selección del personal el $69.2 \%$ de las medianas empresas, un $60 \%$ de las pequeñas y un $51.5 \%$ de las microempresas aplican este proceso en la contratación de personal. El $60 \%$ de las empresas menores a 11 años también lo hacen y $57.4 \%$ de las maduras. Es de notar que esta actividad es realizada por más de la mitad de las empresas sin importar tamaño o antigüedad.

En la capacitación del personal según el tamaño, la mayoría de las empresas lo aplican: las medianas en un $84.6 \%$, las pequeñas en un $71.4 \%$ y las micro en el $72.7 \%$. La misma tendencia se observa con respecto a la antigüedad, la mayoría de las empresas de reciente creación (10 años o menos) realizan capacitación de personal (80\%) y las de 11 años en adelante la llevan a cabo en $72.1 \%$ de los casos.

Para el caso de la promoción del personal, al igual que sucede con otras prácticas de recursos humanos, no se encuentra relación entre el tamaño de las empresas y los porcentajes obtenidos. En este caso, se observa que el grado de utilización de esta práctica está por debajo del $50 \%$; donde $30.8 \%$ de empresas medianas la utilizan, un $28.6 \%$ de pequeñas empresas y un $30.3 \%$ de las microempresas.

En el caso de la antigüedad de las empresas, no existe diferencias significativas entre las empresas jóvenes y las maduras ya que se aplican en $30 \%$ y $29.5 \%$, respectivamente.

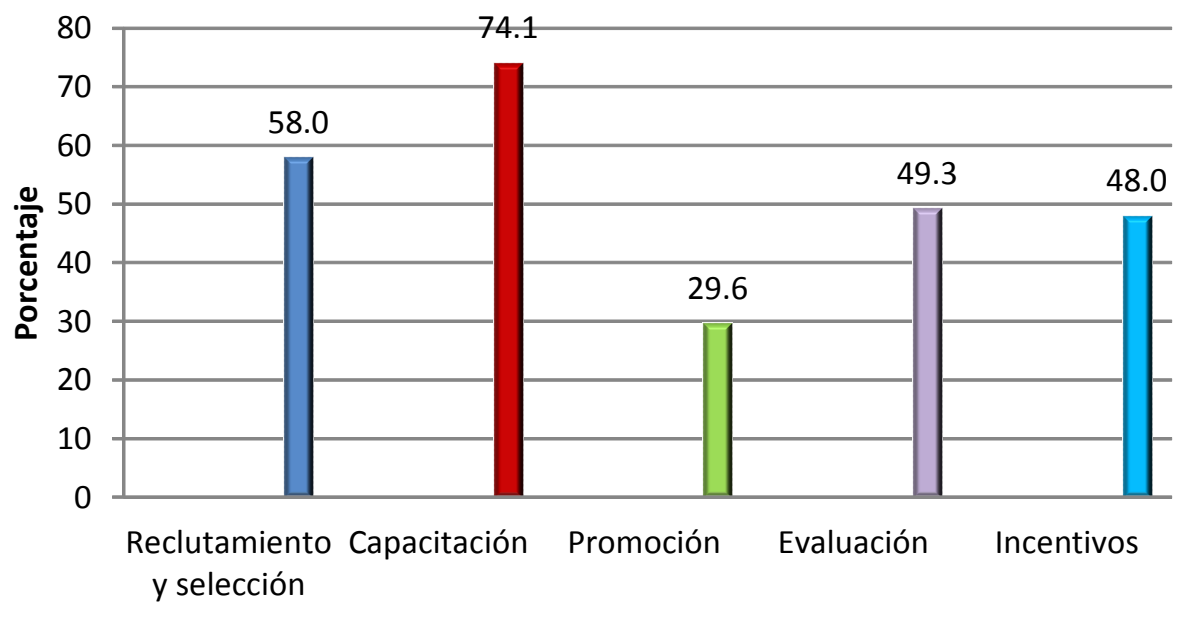

FIGURA 2. PRÁCTICA DE GESTIÓN DE RECURSOS HUMANOS

Fuente: Elaboración propia con datos obtenidos de la base del proyecto de investigación «Análisis comparativo de la competitividad del sector empresarial tabasqueño por ramas de actividad económica» FOMIX-CONACYT - Gobierno del Estado. Clave TAB-2009-C17-120751. 2010 


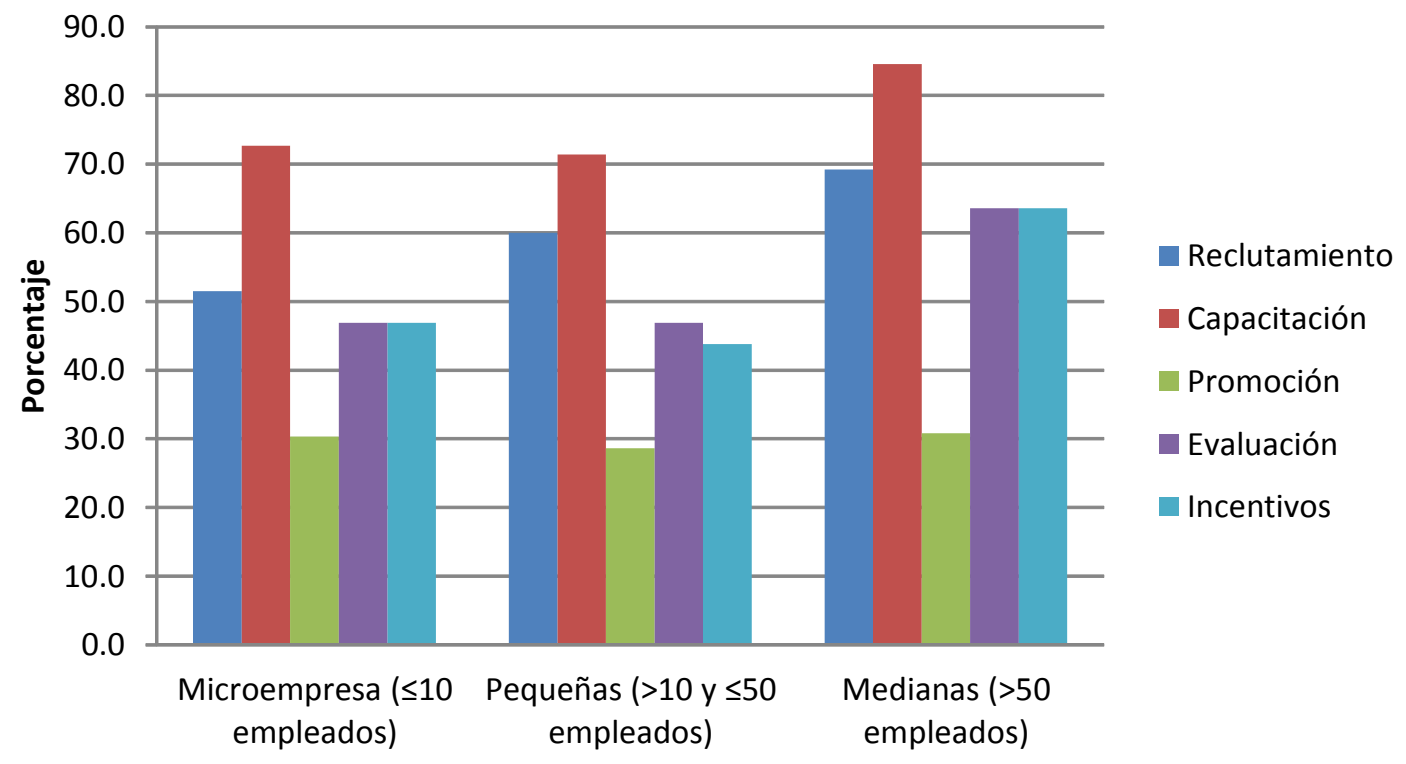

FIGURA 3. PRÁCTICA DE GESTIÓN DE RECURSOS HUMANOS POR TAMAÑO DE EMPRESA

Fuente: Elaboración propia con datos obtenidos de la base del proyecto de investigación «Análisis comparativo de la competitividad del sector empresarial tabasqueño por ramas de actividad económica» FOMIX-CONACYT - Gobierno del Estado. Clave TAB-2009-C17-120751. 2010

Al analizar el uso de la evaluación del desempeño, son las medianas empresas con $63.6 \%$ quienes más la aplican, después encontramos a las micro y pequeñas empresas, ambas con $46.9 \%$. En el caso de la variable antigüedad, esta práctica de recursos humanos se emplea en $50 \%$ y $49.1 \%$ de empresas jóvenes y maduras, respectivamente.

En cuanto a contar con un programa de incentivos, no encontramos diferencias estadísticamente significativas en ninguno de los casos, además, de que las medianas empresas en $63.6 \%$ de los casos realizan esta actividad superando a los porcentajes de los otros tamaños. Además que $44.4 \%$ de las empresas jóvenes aplican el programa de incentivos, en mayor porcentaje encontramos a las maduras con $49.1 \%$.

Si comparamos los resultados de las dos prácticas anteriores podemos notar que en las micro y medianas empresas el porcentaje de empresas que realizan evaluación de desempeño corresponden al porcentaje de las aplican programas de incentivos, sin embargo en las pequeñas empresas no sucede así.

Por otro lado, la promoción de personal es la actividad menos realizada, lo cual refleja que en la mitad de los casos no está incluida dentro de los incentivos para motivar a los empleados.
Se contrastaron cinco variables para aceptar o rechazar la hipótesis nula sobre la gestión de recursos humanos, de las cuales tres se rechazaron (reclutamiento y selección del personal con un resultado de $Z_{c}=-1.93$, promoción del personal con $Z_{c}=-2.33$ y programa de incentivos con $Z_{c}=-1.83$ ) y dos se aceptaron (capacitación del personal con $Z_{c}=-0.32$ y valoración del personal con $Z_{c=}-1.51$ ); por lo tanto se considera que los procesos de gestión de recursos humanos de las MIPYMES de los subsectores comunicaciones y transportes del estado de Tabasco son bajos en Tabasco en comparación con otros estados del país tomados como referencia.

\section{Discusión}

En los resultados obtenidos se puede observar el alto porcentaje de capacitación que existe en las empresas, según la perspectiva de empresario, superior al de reclutamiento y selección de personal y al de promoción de personal, lo cual puede fundamentar el hecho de que el nivel de la gestión de recursos humanos sea poco eficiente en las MIPYMES de los subsectores comunicaciones y transportes del estado ya que como lo señala Hernández \& Martí (2006) una de las finalidades de la selección del personal es establecer políticas y prácticas de obtención del personal idóneo a la organización, así como seleccionar el personal, 